\section{$\underset{\substack{\text { hommes } \\ \text { \& migrations }}}{ }$}

\section{Hommes \& migrations}

Revue française de référence sur les dynamiques

migratoires

$1283 \mid 2010$

Cuisines et dépendances

\title{
France : une anthologie des musiques traditionnelles
}

\section{François Bensignor}

\section{Q OpenEdition \\ Journals}

\section{Édition électronique}

URL : http://journals.openedition.org/hommesmigrations/1019

DOI : 10.4000/hommesmigrations.1019

ISSN : 2262-3353

\section{Éditeur}

Musée national de l'histoire de l'immigration

\section{Édition imprimée}

Date de publication : 1 janvier 2010

Pagination : 190-195

ISBN : 978-2-919040-04-9

ISSN : 1142-852X

\section{Référence électronique}

François Bensignor, "France : une anthologie des musiques traditionnelles», Hommes \& migrations [En ligne], 1283 | 2010, mis en ligne le 29 mai 2013, consulté le 22 septembre 2020. URL : http:// journals.openedition.org/hommesmigrations/1019; DOI : https://doi.org/10.4000/ hommesmigrations.1019

Ce document a été généré automatiquement le 22 septembre 2020.

Tous droits réservés 


\title{
France : une anthologie des musiques traditionnelles
}

\author{
François Bensignor
}

1 L'anthologie des musiques traditionnelles de France, conçue par Guillaume Veillet, est un coffret de $10 \mathrm{CD}$ qui propose un panorama extraordinaire de la diversité des expressions musicales sur tous les territoires qui composent la France d'aujourd'hui. Des Alpes au Berry, de la Bretagne à la Corse, mais aussi de la Guyane à la NouvelleCalédonie et du Québec à la Louisiane, ce recueil de près de 300 airs témoigne de la fabuleuse richesse patrimoniale que constituent les traditions musicales et dansées de tous ces territoires. Leurs ressemblances comme leurs différences contribuent à façonner l'identité d'une culture française dans laquelle s'agrègent les spécificités de groupes sociaux multiples qui ont en partage les institutions de la France et, au-delà, la langue qui les unit et qu'ils défendent jusqu'en dehors de ses frontières.

2 "La plupart des chansons du répertoire traditionnel ont été créées par des auteurs, il y a plusieurs siècles", explique Guillaume Veillet, passionné de ces musiques, collecteur et ancien rédacteur en chef de Trad' Magazine. Elles ont été 'médiatisées' grâce aux 'feuilles volantes', ces partitions qui étaient diffusées dans tout le monde francophone. Et des chanteurs populaires analphabètes, qui se les transmettaient de bouche à oreille, les ont constamment remodelées par une sorte de réécriture collective sur plusieurs générations. Les chansons circulaient avec les populations, au gré des déplacements de travailleurs, sur les bateaux, etc. On retrouve ainsi des versions différentes d'une même chanson en Vendée ou en Haute-Provence, dans le Berry ou le Béarn, tout comme à la Guadeloupe ou au Québec."

3 Cette anthologie des musiques traditionnelles de France propose essentiellement des enregistrements de collectages, souvent inédits. Bien que privilégiant les chanteurs et musiciens non professionnels, l'enchaînement des chansons ou des airs à danser s'écoute avec le plaisir du mélomane. Les livrets offrent un éclairage pertinent sur les traditions, les interprètes, les chansons et leur histoire. Huit albums dressent un panorama des musiques traditionnelles pratiquées dans les régions métropolitaines, débordant vers le Piémont occitan, le Val d'Aoste, la Suisse romande et la Wallonie. 
4 L'un des albums de l'anthologie est consacré au répertoire conservé par les descendants de Français en Amérique du Nord. À partir de 1604, la chanson francophone s'est exportée avec les colons français qui ont commencé à peupler le nord du continent américain. Des airs de Normandie et du Poitou sont encore chantés au Canada ou dans l'ancienne Acadie. Ce répertoire y a parfois été d'autant mieux conservé qu'il a servi de lien culturel identitaire pour des minorités réticentes à s'intégrer dans le monde anglophone. La préservation et la transmission de ces vieilles chansons relèvent en ce cas de l'identité de cultures en résistance.

5 Un autre album est consacré à la richesse des traditions de musiques et de danses à découvrir sur l'ensemble des territoires d'outre-mer. "Pour moi, il était indispensable de montrer la diversité des cultures musicales encore vivantes aux îles Marquises, en Guyane, à Mayotte ou à La Réunion", précise Guillaume Veillet. Il exprime son regret de ne pas avoir pu présenter un aperçu des musiques issues de l'immigration jouées dans l'Hexagone, bien qu'il existe des collectes et des travaux très intéressants dans ce domaine: "Le volume de l'anthologie étant limité à dix albums, j'ai préféré m'abstenir. Mais j'aimerais me lancer un jour dans une anthologie exhaustive des musiques de l'immigration."

\section{Oralité, transmission, archives}

6 C'est au cours des années soixante et soixante-dix que les musiciens issus du mouvement folk se sont intéressés à la mise en valeur des musiques traditionnelles, marqueurs identitaires des cultures régionales. Progressivement s'est mis en place un véritable réseau de collecteurs et de revivalistes passionnés. Au cours des années quatre-vingt et quatre-vingt-dix, celui-ci a pu se structurer, notamment grâce à l'établissement de Centres des musiques et danses traditionnelles, soutenus par l'État et les régions. La vocation de nombre de ces centres en région consiste à favoriser la vitalité des traditions musicales et dansées à travers leur transmission et leur pratique. Si le collectage du patrimoine vivant auprès des anciens et l'archivage de ces collectes relèvent de leurs missions, seuls certains de ces centres en ont fait leur priorité, comme Dastum $^{1}$ en Bretagne. La Fédération des associations de musiques et danses traditionnelles $\left(\mathrm{FAMDT}^{2}\right)$, qui les réunit, a choisi de s'associer à la réalisation de cette anthologie en tant que partenaire ressources. "Une fois le projet accepté par le producteur, Patrick Frémeaux, la FAMDT a fait circuler l'information auprès de ses antennes, explique Guillaume Veillet. Des collecteurs se sont fait connaître et j'ai pu effectuer un tour de France, en privilégiant les centres qui disposent des fonds les plus importants. J'y ai été très bien reçu, par des passionnés qui m'ont conseillé et aiguillé vers les morceaux les plus exemplaires figurant dans leurs archives."

7 Au plan national, deux institutions disposent de fonds d'archives particulièrement intéressants dans le domaine des musiques traditionnelles. Celui de l'ancien musée des Arts et traditions populaires (ATP) à Paris, qui dépend aujourd'hui du musée des Civilisations Europe Méditerranée (MUCEM) ${ }^{3}$, en cours de construction à Marseille. La Bibliothèque nationale de France (BNF) ${ }^{4}$ dispose également de fonds intéressants, dont certains très anciens sont mis en valeur sur son site Internet. "Au musée des ATP, la responsable de la phonothèque, Marie-Barbara Le Gonidec, et Valérie Pasturel, qui y travaillait au moment de mes recherches, m'ont ouvert l'accès à l'ensemble des fonds, dit Guillaume Veillet. J'ai passé plusieurs semaines à écouter des centaines d'enregistrements. Catherine Perrier l'avait fait de manière exhaustive pour la période allant de 1939 à 1962. Et j'ai poursuivi 
l'inventaire de la période postérieure à 1962, un travail extrêmement enrichissant. J'ai également pu rencontrer de nombreux collecteurs individuels qui travaillent indépendamment des réseaux institutionnels et associatifs. Par exemple, alors que j'avais des difficultés à trouver des enregistrements pour l'Alsace, j'ai rencontré un instituteur, Jean-Marie Ehret, qui a fait de très intéressantes collectes dans un canton frontalier avec les Vosges."

$8 \quad$ Les musiques traditionnelles se différencient de la chanson populaire française en ceci qu'elles sont chantées et jouées par des interprètes dits "de tradition", qui ne font pas une profession de la musique. Les musiciens professionnels revivalistes, s'ils ne sont pas les interprètes de cette anthologie, sont pourtant à la base de nombreux enregistrements qu'elle contient. Bien souvent, ce sont eux qui ont réalisé les collectages ou sont à l'origine des événements revivalistes, comme les fêtes du chant, au cours desquels les chanteurs et musiciens traditionnels ont pu être enregistrés. Tous les enregistrements publiés dans cette anthologie sont libres de droits. Certains producteurs de disques commercialisés ont donné leur aimable autorisation pour que des morceaux extraits de leurs catalogues puissent y figurer. Mais pour la majorité, il s'agit d'enregistrements appartenant soit au domaine public, soit à des associations de musiques traditionnelles qui n'exigent pas de droits pour qu'ils figurent dans cette publication.

\section{La France d'outre-mer}

"Dans ce disque, j'ai voulu montrer la richesse des expressions locales anciennes, mais aussi l'apport des migrations, des mélanges de populations qui ont abouti à une créolisation des cultures, explique Guillaume Veillet. L'outre-mer est une bonne vitrine pour l'actualité de la collecte. Tant pour le répertoire local que pour le répertoire francophone qui y subsiste. Dans certains cas, on constate que ces territoires ont été des lieux de préservation pour certains répertoires régionaux, qui se sont perdus dans les régions d'origine."

10 Les fonds du musée des ATP s'avèrent une source extrêmement riche sur le plan des collectes concernant l'outre-mer. Les ethnologues Claudie Marcel-Dubois et Marguerite Pichonnet-Andral ont en effet consacré leurs dernières années en poste aux ATP presque exclusivement à la France des Dom Tom. "Dès 1971, elles ont mené des travaux en Martinique et en Guadeloupe, dit Guillaume Veillet. À Saint-Barthélemy, elles ont fait des collectes particulièrement intéressantes. Peuplée essentiellement de descendants de colons normands, bretons, poitevins, saintongeais et angevins, l'île abrite un répertoire très riche de chansons traditionnelles françaises, auquel l'ethnomusicologue américain Alan Lomax s'était intéressé dès 1962. En 1978-79, elles ont fait une enquête passionnante dans les Mascareignes : La Réunion, Maurice et Rodrigues. Elles sont probablement parmi les premières métropolitaines à s'être intéressées à Firmin Viry et Lo Rwa Kaf, deux des figures les plus emblématiques du maloya traditionnel de La Réunion. Ces fonds consacrés à la Martinique et à La Réunion étaient jusqu'à présent inédits."

11 En menant ses recherches sur les musiques d'outre-mer, Guillaume Veillet constate que les interlocuteurs se sont multipliés sur le terrain au cours des dix dernières années. "Il existe beaucoup de collectes récentes sur de très nombreux terrains, explique-t-il. Pour Mayotte, par exemple, pendant longtemps on n'avait pas d'enregistrements. Or, depuis quelque temps, les archives se structurent. Une campagne de collecte a été lancée récemment par la jeune ethnologue Fanny Précourt pour le Pôle régional de musiques actuelles de La Réunion (PRMA). Elle m'a beaucoup aidé pour les musiques de l'océan Indien." Le PRMA ${ }^{(5)}$ fait figure de 
pionnier. Afin d'assurer sa mission de sauvegarde du patrimoine musical de l'île mais aussi des autres îles du sud-ouest de l'océan Indien et de restituer au public le fruit des collectes effectuées, le Pôle s'est doté en 1998 d'un label de disque, Takamba, dont le catalogue propose aujourd'hui une quinzaine d'albums.

En Guyane, le Centre d'archives des documents ethnographiques de la Guyane (Cadeg) ${ }^{\left({ }^{6}\right)}$ s'est mis en place en 2006. Il fonctionne depuis deux ans au sein de l'université des Antilles et de la Guyane autour des missions de repérage, d'archivage et de traitement des données du patrimoine immatériel de la Guyane. "Je me suis servi des enquêtes récentes qu'a menées Apollinaire Anakesa sur les Bushinengue, pour lesquels il existait très peu d'enregistrements", dit Guillaume Veillet.

En Guadeloupe, il a pu bénéficier de l'aide du Centre des musiques et danses traditionnelles et populaires Rèpriz ${ }^{7}$, dont le bureau a ouvert en janvier 2010 à Pointeà-Pitre. Parmi ses missions figure la "sauvegarde du patrimoine musical ancien et actuel de la Guadeloupe à travers des collectes, recherches scientifiques, réalisations de documentations, animations, publications destinées aussi bien aux spécialistes, artistes, et enseignants qu'au public le plus large."

14 "Dans ma quête des musiques d'outre-mer, j'ai été confronté à deux types de réactions, dit Guillaume Veillet. Certains de mes interlocuteurs trouvaient dommage que le travail soit confié à un métropolitain. Il est vrai que j'agissais en candide pour ces terrains que je ne connaissais pas. Mais d'autres trouvaient formidable l'idée de présenter sur un même disque toute la richesse de ces musiques provenant de lieux géographiques extrêmement éloignés les uns des autres. D'autant que ce travail n'avait encore jamais été fait."

\section{Français d'Amérique}

Exploré dès les années soixante par les revivalistes, le fonds francophone d'Amérique du Nord est mieux connu. Mais il regorge d'étonnants témoignages historiques, comme l'explique Guillaume Veillet: "En faisant mes recherches pour l'anthologie, j'ai découvert des chansons transmises par les 'coureurs de bois', les fameux trappeurs français qui avaient une femme à Québec, une autre à Saint-Louis et une ou deux dans les tribus amérindiennes avec lesquelles ils commerçaient. L'exemple présenté dans l'anthologie est une savoureuse chanson à boire interprétée quasi phonétiquement par Lawrence Keplin, descendant d'indiens Cree. Il n'est pas francophone, $d u$ fait de son éducation scolaire, mais continue à chanter en français les chansons qu'il tient de son grand-père, qui lui-même les tenait de lointains aïeux coureurs de bois français."

16 La richesse du répertoire des Français d'Amérique est due principalement aux influences culturelles des autres populations migrantes. "Au Canada, par exemple, la musique des anglophones et des francophones présente peu de différences, souligne Guillaume Veillet. Les influences anglo-saxonne et irlandaise y sont prédominantes. En l'occurrence, la créolisation s'est faite plus généralement à partir d'éléments tirés des traditions musicales véhiculées par les colons. En Louisiane, on observe un brassage extraordinaire d'influences, entre les Espagnols, les Français, les anciens esclaves venus d'Haïti, les anglophones et les Allemands qui introduisirent l'accordéon. Alma Barthélémy, qui chante une version de La claire fontaine, incarne ce brassage. Elle est un quart Espagnole, un quart Créole française, un quart Amérindienne et enfin descendante d'esclaves venus d'Haiti. Illettrée, elle était néanmoins 
parfaitement francophone et connaissait des centaines de chansons. Dans son interprétation, on peut discerner toutes les influences qui se sont exercées sur la musique de la Louisiane."

En Amérique du Nord, les fonds d'archives, qui se sont constitués il y a déjà longtemps, sont assez conséquents. "Pour le Québec, j'ai travaillé à partir des Archives de folklore et d'ethnologie de l'université Laval"), qui sont assez anciennes, dit Guillaume Veillet. Pour l'Acadie, le Centre d'études acadiennes de Moncton") possède des fonds réunis par des militants culturels, collecteurs enthousiastes et souvent universitaires, qui ont fait de ce centre un conservatoire de la culture francophone. Quelques collecteurs individuels de mes connaissances ont également été sollicités, comme Jean-Pierre Bruneau, qui a fait de superbes enregistrements dans les années soixante-dix avec tous les grands noms de la musique cajun. Roger Mason, musicien américain vivant en France, a fait des enquêtes passionnantes en Louisiane, comme membre du musée des ATP. Le Centre franco-ontarien de folklore ${ }^{10)}$ a également été l'un de nos partenaires enthousiastes. Et pour l'anecdote, j'ai tenu à publier deux chansons chantées en anglais, français et wallon, provenant de la communauté wallonne du Wisconsin."

\section{NOTES}

1. Dastum: www.dastum.net

2. FAMDT: www.famdt.com

3. MUCEM: www.musee-europemediterranee.org

4. BNF : www.bnf.fr

5. Pôle régional de musiques actuelles de la Réunion (PRMA) : www.runmuzik.fr

6. Centre d'archives des documents ethnograhiques de la Guyane (Cadeg) : www.cadeg.fr

7. Centre des musiques et danses traditionnelles et populaires Rèpriz : www.repriz-cmdtp.org

8. Archives de folklore et d'ethnologie de l'Université Laval : www.archives.ulaval.ca

9. Centre d'études acadiennes de Moncton: www0.umoncton.ca/etudeacadiennes/centre/ folk1.html

10. Centre franco-ontarien de folklore : www.cfof.on.ca

\section{RÉSUMÉS}

Le thème de l'identité nationale a fait resurgir certaines notions nauséabondes empruntées aux heures les moins glorieuses du passé de la France. Ces dérives auraient sans doute pu être évitées si l'on avait daigné s'intéresser à la première anthologie consacrée aux musiques traditionnelles de France, publiée à l'automne 2009 par l'éditeur Frémeaux \& Associés. 